\title{
Qualidade de vida e aspectos sociodemográficos de doentes renais crônicos
}

\author{
Quality of life and sociodemographic aspects of chronic kidney patients
}

Calidad de vida y aspectos sociodemográficos de pacientes renales crónicos.

\begin{abstract}
Silas Alves da Silva ${ }^{1 *}$, Filipe Melo da Silva ${ }^{1}$, Antônio Eduardo Osório Cavalcante ${ }^{1}$, Emanuel Thomaz de Aquino Oliveira ${ }^{1}$, Matheus Halex Ferreira de Matos ${ }^{1}$, Anando Rodrigues de Carvalho ${ }^{1}$, Ana Christina de Sousa Baldoino², Lívia dos Santos Lopes Assis ${ }^{1}$, Giovanna de Oliveira Libório Dourado ${ }^{1}$, Jailson Alberto Rodrigues ${ }^{1}$.
\end{abstract}

\section{RESUMO}

Objetivo: Descrever a qualidade de vida e aspectos sociodemográficos de doentes renais crônicos em terapêutica hemodialítica em um município de médio porte do Nordeste Brasileiro. Métodos: Estudo descritivo, transversal, realizado com 21 pacientes portadores de doença renal crônica submetidos à hemodiálise. Utilizou-se a versão curta do instrumento Kidney Disease and Quality of Life Short Form, que avalia qualidade de vida. O Comitê de Ética e pesquisa aprovou a pesquisa com parecer № 3.683.252. Resultados: Os mais acometidos pela doença são homens $(p=0,078)$; solteiros $(p=0,006)$; que ganham entre 1 - 3 salários mínimos $(p=0,011)$; aposentados $(p=0,011)$ e católicos $(p=0,003)$. Às médias dos escores: carga da doença renal $(36,3)$, efeitos da doença renal na vida diária $(40,0)$, o componente físico $(39,5)$ e componente mental $(45,1)$, espelham escore geral médio de qualidade de vida ruim. Porém, a dimensão de sintomas da doença renal $(64,8)$ destaca escore moderado para qualidade de vida. Conclusão: Reforça-se que é preciso haver conhecimento acerca do universo do paciente portador da doença renal crônica, assim, podem ser promovidas mudanças, como maior participação dos familiares e maior atenção da equipe no cuidado dos usuários.

Palavras-chaves: Doença renal crônica, Hemodiálise, Qualidade de Vida.

\begin{abstract}
Objective: To describe the quality of life and sociodemographic aspects of chronic kidney patients undergoing hemodialysis in a medium-sized municipality in Northeast Brazil. Methods: A descriptive, cross-sectional study conducted with 21 patients with chronic kidney disease undergoing hemodialysis. The short version of the Kidney Disease and Quality of Life Short Form instrument was used, which assesses quality of life. The Ethics and Research Committee approved the research with opinion No. 3,683,252. Results: The most affected by the disease are men $(p=0.078)$; singles $(p=0.006)$; earning between $1-3$ minimum wages $(p=0.011)$; retired $(p=0.011)$ and catholic $(p=0.003)$. Mean scores: kidney disease burden (36.3), effects of kidney disease on daily life (40.0), the physical component (39.5) and mental component (45.1), mirror the average general score of bad quality of life. However, the dimension of symptoms of kidney disease (64.8) highlights a moderate score for quality of life. Conclusion: It is reinforced that it is necessary to have knowledge about the universe of the patient with chronic kidney disease, thus, changes can be promoted, such as greater participation of family members and greater attention of the team in the care of users.
\end{abstract}

Keywords: Chronic kidney disease, Hemodialysis, Quality of life.

\footnotetext{
1 Universidade Federal do Piauí (UFPI), Floriano - PI. *E-mail: silasalvesilva@yahoo.com.br
}

2 Universidade Estadual do Piauí (UESPI), Floriano - PI. 


\section{RESUMEN}

Objetivo: Describir la calidad de vida y aspectos sociodemográficos de los pacientes renales crónicos en hemodiálisis en un municipio de tamaño medio del Nordeste de Brasil. Métodos: Estudio descriptivo, transversal, realizado con 21 pacientes con enfermedad renal crónica en hemodiálisis. Se utilizó la versión corta del instrumento Kidney Disease and Quality of Life Short Form que evalúa la calidad de vida. El Comité de Ética e Investigación aprobó la investigación con el dictamen No. 3.683.252. Resultados: Los más afectados por la enfermedad son los hombres $(p=0,078)$; solteros $(p=0,006)$; ganar entre 1 y 3 salarios mínimos $(p=0,011)$; jubilados $(p=0,011)$ y católicos $(p=0,003)$. Puntuaciones medias: carga de enfermedad renal $(36,3)$, efectos de la enfermedad renal en la vida diaria $(40,0)$, el componente físico $(39,5)$ y el componente mental $(45,1)$, reflejan la puntuación general media de mala calidad de vida. Sin embargo, la dimensión de síntomas de enfermedad renal $(64,8)$ destaca una puntuación moderada para la calidad de vida. Conclusion: Se refuerza que es necesario tener conocimiento sobre el universo del paciente con enfermedad renal crónica, así, se pueden promover cambios, como una mayor participación de los familiares y una mayor atención del equipo en la atención a los usuarios.

Palabras clave: Enfermedad renal crónica, Hemodiálisis, Calidad de vida.

\section{INTRODUÇÃO}

A doença renal crônica (DRC) é definida como a perda permanente das funções renais, alterando a homeostasia corporal humana, e, caso não haja uma terapia renal substitutiva (TRS), pode levar ao óbito (MIYAHIRA CK, et al., 2016).

À vista disso, na última década, houve um aumento no número de casos de DRC em diferentes contextos, associados ao envelhecimento e melhora na expectativa de vida. Por isso, há o aumento de pessoas portadoras de doenças crônicas, como a hipertensão arterial sistêmica (HAS) e diabetes, sendo as principais causas da DRC (RIBEIRO KRA, 2016).

Acerca dos métodos para terapia dialítica, a hemodiálise é o mais aplicado, tratando-se de um processo intermitente, que ocorre usualmente três vezes na semana, durando 4 horas por sessão (VENTURA J, et al., 2018). Desse modo, o usuário renal crônico convive constantemente com o processo doloroso da terapia, bem como suas limitações, afetando sua qualidade de vida (QV) (SANTOS SBP, et al., 2017).

Assim, a DRC, somada ao tratamento hemodialítico, refletem mudanças que abalam negativamente a qualidade de vida (QV) tanto do seu portado quanto dos familiares (RIBEIRO KRA, 2016). Além disso, o tratamento desencadeia conflitos emocionais, vivências angustiantes, traumatizantes e complexas, que são capazes de mudar toda a vida do doente (CRUZ MRF, et al., 2016).

Nesse sentido, qualidade de vida (QV) é apresentado com uma ampla dimensionalidade, constituída por aspectos físicos, psicológicos, sociais e ambientais. Analisa-se a capacidade de o indivíduo viver em bemestar físico, psíquico e social e não somente em ausência de doença e enfermidade (OLIVEIRA APB, et al., 2016). Dessa forma, entende-se QV como a percepção do indivíduo de sua posição na vida, no contexto da cultura e sistema de valores nos quais ele vive e em relação aos seus objetivos, expectativas, padrões e preocupações (PEREIRA RMP, et al., 2017).

Nesse aspecto, apesar do crescente número de pesquisas sobre o tema, ainda é notória a necessidade por mais estudos que contemplem a QV de pessoas portadores da DRC e sua associação com as características clinicas e sociodemográficos. Sendo possível, a partir disso, haver orientações e intervenções que objetivam o melhoramento do nível de saúde e da QV dessa população (OLIVEIRA APB, et al., 2016).

Dessa forma, o presente estudo tem por objetivo descrever a qualidade de vida e aspectos sociodemográficos de doentes renais crônicos em terapêutica hemodialítica em um município de médio porte do Nordeste brasileiro. 


\section{MÉTODOS}

Trata-se de um estudo transversal, realizado com pacientes portadores de DRC submetidos à hemodiálise. Como cenário, elegeu-se um município de médio porte do nordeste brasileiro. A amostra deu-se por conveniência, os participantes do estudo foram abordados entre os meses de janeiro e março de 2020, com duração de aproximadamente, 30 minutos em domicílio.

Os participantes do estudo foram pessoas com DRC, em tratamento hemodialítico e amparados pelas Unidades Básicas de Saúde da Atenção Básica. A priori, a Secretaria Municipal de Saúde (SMS) disponibilizou um total de 41 endereços de usuários. Ao buscar os sujeitos, encontrou-se 3 casos de óbitos, 2 altas do tratamento, 15 mudaram de endereço ou cidade sem atualização dos dados. Dessa forma, foram incluídas 21 pessoas no estudo.

Foram incluídas no estudo usuários com idade superior a 18 anos, residentes no município e aqueles que tinham capacidade cognitiva de responder ao instrumento. A coleta de dados deu-se pela caracterização do perfil dos clientes, em que são coletados dados sociodemográficos e clínicos. Além da avaliação da QV, a partir do qual se utilizou o Kidney Disease and Quality of Life Short Form (KDQOL-36 ${ }^{\mathrm{TM}}$, desenvolvido para avaliar a QV de portadores de DRC em tratamento hemodialítico).

O KDQOL-36 тм inclui o SF-12: resumo do componente físico e resumo do componente mental como núcleo genérico. Mais o ônus da doença renal: carga da doença renal, sintomas/problemas e efeitos da DRC na vida diária. Os itens estão subdivididos na sequência: Itens 1-12: SF-12; Itens 13-16: Carga de doença renal; Itens 17-28: Sintomas / problemas; Itens 29-36: Efeitos da doença renal. Os dados coletados sobre a QV foram organizados no programa Excel for Windows (Microsoft), através de uma ferramenta desenvolvida pelo KDQOL Working Group. Alimentando o banco de dados do estudo exportado para o programa SPSS, Versão 20.0.

A partir disso, foram aplicadas técnicas de estatística descritiva e inferencial. Tomou-se como referência, o nível alfa de significância estatística 0,05 para realização das inferências. Depois disso, foram realizados métodos descritivos e, as inferências estatísticas foram norteadas a partir da análise de $T$-Student para as variáveis de natureza intervalar. Foi verificada a correlação de Spearman, visto a natureza de não normalidade dos dados a serem obtidos.

Todos os participantes do estudo assinaram o Termo de Consentimento Livre e Esclarecido (TCLE). Além disso, a proposta de estudo foi encaminhada ao Comitê de Ética em Pesquisa (CEP) da Universidade Federal do Piauí (UFPI), CAAE: 19750519.4.0000.5214, e, após apreciação, foi efetivada a pesquisa com parecer $n^{\circ}$ 3.683.252.

\section{RESULTADOS}

Em relação aos dados sociodemográficos, a amostra foi composta por 21 pessoas portadoras de DRC e que realizam tratamento hemodialítico, destes, 15 (71,4\%) são do sexo masculino e, 11 (52,4\%) afirmaram ser solteiros (Tabela 1). A faixa etária entre 60 e 79 anos foi a prevalente, com 8 usuários $(31,1 \%)$, e a maioria dos participantes moram com seus cônjuges $(33,3 \%)$. Com relação à escolaridade, aqueles com ensino fundamental incompleto foram os mais presentes $(38,1 \%)$.

A maior parte declarou ser católica $(57,1 \%)$, sendo predominantemente aposentados $(47,6 \%)$. Os que não trabalham e não estudam somam $38,1 \%$ e, a maioria dos DRC tem uma renda entre 1 e 3 salários mínimos $(47,6 \%)$. Os que recebem um salário mínimo aparecem em seguida contando $38,1 \%$ dos participantes (Tabela 1).

Houve sustentação estatística evidenciando que as variáveis, cor/raça $(p=0,368)$, faixa etária $(p=0,502)$, escolaridade $(p=0,165)$, com quem mora $(p=0,423)$, causa da DRC $(p=0,406)$ e tempo de tratamento $(p=$ 312), neste estudo, os indivíduos que se enquadrem nessas características das variáveis acima, todos são acometidos pela DRC de igual modo. Ressalta-se que a variável sexo $(p=0,078)$ apresentou uma relativa significância, apontando que os homens, no nosso estudo, são os mais inquiridos pela DRC. 
De outro modo, as variáveis estado civil $(p=0,006)$, renda $(p=0,011)$, atividade/fonte de renda $(p=0,011)$ e religião $(p=0,003)$ obtiveram níveis de significância que indicam haver uma diferença estatística na dinâmica de acometimento populacional da DRC nos usuários que nelas se enquadram. Há, portanto, evidências estatísticas para se afirmar, que os mais acometidos pela DRC são solteiros; os que ganham entre 1 e 3 salários mínimos, aposentados e católicos.

Tabela 1 - Dados sociodemográficos dos portadores de doença renal crônica. $\mathrm{N}=21$.

\begin{tabular}{|c|c|c|c|}
\hline Variáveis & Descrição & $\mathrm{n}(\%)$ & p-valor \\
\hline \multirow{2}{*}{ Sexo } & Feminino & $06(28,6)$ & \multirow{2}{*}{0,078} \\
\hline & Masculino & $15(71,4)$ & \\
\hline \multirow{4}{*}{ Estado Civil } & Solteiro & $11(52,4)$ & \multirow{4}{*}{0,006} \\
\hline & Casado & $07(33,3)$ & \\
\hline & Viúvo & $02(9,5)$ & \\
\hline & Divorciado & $01(4,8)$ & \\
\hline \multirow{3}{*}{ Raça/Cor } & Branco & $04(19,0)$ & \multirow{3}{*}{0,368} \\
\hline & Pardo & $09(42,9)$ & \\
\hline & Preto & $08(38,1)$ & \\
\hline \multirow{4}{*}{ Faixa Etária } & $18-39$ anos & $05(23,8)$ & \multirow{4}{*}{0,502} \\
\hline & $40-59$ anos & $07(33,3)$ & \\
\hline & $60-79$ anos & $08(38,1)$ & \\
\hline & 80 anos ou mais & $01(4,8)$ & \\
\hline \multirow{4}{*}{ Escolaridade } & Fundamental completo & $06(28,6)$ & \multirow{4}{*}{0,165} \\
\hline & Fundamental incompleto & $08(38,1)$ & \\
\hline & Ensino médio & $06(28,6)$ & \\
\hline & Ensino Superior & $01(4,8)$ & \\
\hline \multirow{3}{*}{ Renda* $^{*}$} & Até 1 & $08(38,1)$ & \multirow{3}{*}{0,011} \\
\hline & Entre $1-3$ & $12(47,6)$ & \\
\hline & Entre $3-5$ & $01(14,3)$ & \\
\hline \multirow{4}{*}{ Atividade / Fonte de Renda } & Aposentado & $10(47,6)$ & \multirow{4}{*}{0,011} \\
\hline & Trabalha & $01(4,8)$ & \\
\hline & Estuda & $02(9,5)$ & \\
\hline & Não trabalha e não estuda & $8(38,1)$ & \\
\hline \multirow{6}{*}{ Com quem mora } & Cônjuge & $07(33,3)$ & \multirow{6}{*}{0,423} \\
\hline & Familiares & $04(19,0)$ & \\
\hline & Pais & $03(14,3)$ & \\
\hline & Amigos & $01(4,8)$ & \\
\hline & Sozinho & $03(14,3)$ & \\
\hline & Filhos & $03(14,3)$ & \\
\hline \multirow{4}{*}{ Religião } & Católico & $12(57,1)$ & \multirow{4}{*}{0,003} \\
\hline & Evangélico & $06(28,6)$ & \\
\hline & Agnóstico & $01(4,8)$ & \\
\hline & Não declarado & $02(9,5)$ & \\
\hline
\end{tabular}

Legenda: *Em salário mínimo.

Fonte: Silva SA, et al., 2020.

A Hipertensão Arterial Sistêmica (HAS) foi à causa mais relatada $(28,6 \%)$ como doença de base para o surgimento da DRC, acompanhada do Diabetes Mellitus (19,0\%) e glomerulonefrites (19,0\%). No tocante ao tempo de tratamento em hemodiálise, a maior porção é daqueles usuários entre 2 e 5 anos de tratamento $(38,1 \%)$ (Tabela 2). 
Tabela 2 - História clínica dos portadores de doença renal crônica, N = 21 .

\begin{tabular}{clcc}
\hline \multicolumn{1}{c}{ Variável } & \multicolumn{1}{c}{ Descrição } & $\mathbf{n}(\%)$ & p-valor \\
\hline & Diabetes & $04(19,0)$ & \\
Causa da Doença & Hipertensão arterial sistêmica (HAS) & $06(28,6)$ & \\
Renal Crônica & Glomerulonefrites & $04(19,0)$ & 0,406 \\
& Rins policísticos & $01(4,8)$ & \\
& Outras causas & $06(28,6)$ & \\
\hline \multirow{2}{*}{ Tempo de Tratamento } & $1-2$ anos & $06(28,6)$ & \\
& $2-5$ anos & $08(38,1)$ & 0,312 \\
& $5-10$ anos & $05(23,8)$ & \\
\hline
\end{tabular}

Fonte: Silva SA, et al., 2020.

Com relação à patologia de base para o surgimento da DRC, neste estudo, não houve evidência estatística que embase a associação de alguma das doenças descritas acometendo mais a este perfil de usuários que outro. Entretanto, sabe-se que isso está em desacordo com a maioria dos estudos recentes (Tabela 2).

Os escores gerais de todas as dimensões são apresentados na Tabela 3, permitem inferir que as médias gerais de escores mais baixos são verificados nas dimensões: carga da doença renal $(36,3)$, efeitos da doença renal na vida diária $(40,0)$ e resumo de componente físico $(39,5)$. Estas compõem o KDQOL-36 ${ }^{\mathrm{TM}}$, tanto as dimensões genéricas quanto específicas.

Tabela 3 - Escores das dimensões genéricas e específicas do Kidney Disease and Quality of Life Short Form (KDQOL-36 ${ }^{\mathrm{TM}}$ ) dos pacientes em tratamento hemodialítico. $\mathrm{N}=21$.

\begin{tabular}{lllll}
\hline Item do KDQOL-36'M & Média & Mediana & Desvio Padrão & Amplitude \\
\hline Carga da doença renal (4) & 36,3 & 43,7 & 20,4 & $6,2-68,7$ \\
Sintomas/problemas da doença renal (12) & 64,8 & 68,7 & 16,5 & $29,1-89,5$ \\
Efeitos da doença renal na vida diária (8) & 40,0 & 37,5 & 16,5 & $18,7-71,8$ \\
Resumo de Componente Físico & 39,5 & 39,4 & 7,2 & $24,7-51,9$ \\
Resumo de Componente Mental & 45,1 & 45,3 & 8,2 & $28,8-60,8$ \\
\hline
\end{tabular}

Fonte: Silva SA, et al., 2020.

Em relação às medianas dos escores trazidos na Tabela 3, os mais baixos correspondem aos efeitos da DRC $(37,5)$ e componente físico $(39,4)$. Já nas amplitudes, o escore mais baixo de um usuário foi registrada na carga da DRC (6,2); seguido dos efeitos da DRC $(18,7)$, a mais alta oi registrada nos sintomas/problemas da doença renal. Em relação ao desvio padrão, os resumos de componentes físicos $(7,2)$ e mentais $(8,2)$ apontaram os mais baixos.

No tocante às médias gerais de todas as dimensões do $\mathrm{KDQOL}-36^{\mathrm{TM}}$, relacionando com as faixas do quartis, observam-se que a carga da doença renal $(36,3)$, efeitos da doença renal na vida diária $(40,0)$, resumo do componente físico $(39,5)$ e resumo de componente mental $(45,1)$, espelham um escore geral médio para uma QV ruim. Porém, a dimensão de sintomas ou problemas da DRC $(64,8)$ destaca-se com média geral de escore moderado para QV.

Com relação às interferências entre as dimensões dos componentes da qualidade de vida avaliados através do KDQOL - 36, a matriz de significância trazida na Tabela 4 apresenta as significâncias estatísticas dessas relações. Foram testadas as associações de todas as dimensões gerais do instrumento de Avalição de qualidade de vida, a partir da correlação de Spearman. 
Tabela 4 - Matriz de significância ( $p$ - valor) das influências entre as dimensões do KDQOL - 36. $N=21$.

\begin{tabular}{cccccc}
\hline Dimensões & $\begin{array}{c}\text { Componentes } \\
\text { físicos }\end{array}$ & $\begin{array}{c}\text { Componentes } \\
\text { mentais }\end{array}$ & $\begin{array}{c}\text { Sintomas } \\
\text { da DRC }\end{array}$ & $\begin{array}{c}\text { Efeitos da DRC } \\
\text { na vida diária }\end{array}$ & $\begin{array}{c}\text { Carga } \\
\text { da DRC }\end{array}$ \\
\hline Componentes físicos & 0,000 & 0,020 & 0,000 & 0,887 & 0,442 \\
Componentes mentais & & 0,000 & 0,000 & 0,201 & 0,052 \\
Sintomas da DRC & & & 0,000 & 0,000 & 0,000 \\
Efeitos da DRC na vida & & & 0,000 & 0.431 \\
diária & & & & 0,000 \\
Carga da DRC & & & & & \\
\hline
\end{tabular}

Fonte: Silva SA, et al., 2020.

É possível notar, a partir da matriz de significância (Tabela 4), que houve correlações estatísticas entre as dimensões dos componentes físicos e mentais $(p<0,020)$; componentes físicos e sintomas da $D R C(p<0,000)$; componentes mentais e sintomas da DRC $(p<0,000)$; componentes mentais e carga da DRC $(p<0,052)$; sintomas da DRC e efeitos da DRC na vida diária $(p<0,000)$; sintomas da DRC e carga da DRC $(p<0,000)$. Tais dimensões mostraram associação de interferências entres as mesmas, isto é, uma influência diretamente a outra.

\section{DISCUSSÃo}

O perfil dos participantes era de homens, solteiros, entre 60 e 79 anos, moravam com familiares ou cônjuge, baixo nível de escolaridade, religião católica, aposentados e com renda entre 1 a 3 salários mínimos. Houve prevalência de doentes renais crônicos do sexo masculino, embora não tenha se verificado significância estatística ao nível 0,05 , mas a 0,10 que também é um importante balizador e isso, possibilita afirmar que os homens são os mais acometidos pela DRC neste recorte.

No tocante à caracterização de pessoas com DRC, estudos mostram perfis variáveis. Em um estudo realizado com pacientes renais crônicos de um hospital de nefrologia, no Rio de Janeiro, identificaram que a maioria dos doentes eram do sexo feminino (54,3\%) (MENEZES HF, et al., 2017). Conforme o inquérito Brasileiro de Diálise realizado pela Sociedade Brasileira de Nefrologia, $58,0 \%$ dos portadores eram do sexo masculino (MARQUES VR, et al., 2016).

Nesse estudo, existe significância estatística que evidencia que os solteiros são os mais acometidos pela DRC. Em São Paulo, estudo, que abordou 386 doentes renais crônicos em um hospital encontrou que a maioria eram casados $(59,8 \%)$ (MONTEIRO MAC, et al., 2018).

O estudo evidencia, em relação à raça/cor, que, estatisticamente não houve significância para a hipótese levantada de detrimento da cor predominante (Pardos). O que indica uma distribuição normal e igual da DRC dentre as raças. Entretanto, há evidências que indicam maior tendência de que os pacientes pardos sejam os mais acometidos pela DRC (OLIVEIRA APB, et al., 2016).

Em relação às faixas etárias dos usuários deste estudo, percebe-se uma uniformidade de distribuição. Isto é, não houve correlação estatística entre a ocorrência da DRC e uma faixa de idade específica. Outros estudos apresentam associação entre idade e DRC, destacando a ocorrência em pessoas acima dos 40 anos e em idosos (GOLÇALVES FA, et al., 2015; SORTORI A, et al., 2017).

No que tange à escolaridade, não houve significância estatística demonstrando relação com a DRC. Resultado semelhante encontrado em um estudo com pessoas em tratamento de hemodiálise em São Paulo (GESUALDO GB, et al., 2016). Em contraste, um estudo realizado com 80 pacientes, afim de identificarem o impacto do cronotipo na QV dos doentes renais crônicos, evidenciou que há uma maior relação entre os usuários e a baixa escolaridade (OTONI A, et al., 2019). 
Concernente à renda dos portadores, a pesquisa denota que há significativa associação da DRC com aqueles que têm renda de 1 a 3 salários mínimos. Achados semelhante a pesquisas realizadas, as quais identificaram que a maioria dos doentes renais $(67,3 \%)$ tinha a mesma faixa de renda (ASLAN I, 2018).

Ademais, um estudo com 105 doentes renais crônicos em uma clínica de tratamento hemodialítico encontrou achados diferentes. Desvendando na pesquisa que a maioria dos clientes $(83,8 \%)$ viviam com uma renda não superior a 1 salário mínimo (BEBER GC, et al., 2017). Além disso, o fato de boa parte dos participantes possuírem renda de até um salário mínimo é decorrente de benefícios cedidos pelos governos, por não conseguirem desenvolver atividades devido às limitações físicas e também do tempo dispensado ao tratamento (PEIPERT JD, et al., 2019).

Pode-se inferir que existe uma relação que se retroalimenta entre baixa renda e DRC. O tratamento hemodialítico impõe uma rotina que interfere diretamente na atividade laboral, visto que é necessário comparecer regularmente aos serviços de saúde, o que impossibilita manter um turno diário de trabalho. Assim, essas pessoas tem a renda prejudicada em virtude da dificuldade em manter um emprego formal. No caso de pessoas em idade escolar ou jovens interfere ainda na participação em cursos presenciais. Esses aspectos perpetuam a situação de pobreza.

Acerca da coabitação, associação com nenhuma variável exposta, a não ser a indicação de uma tendência percentual para aqueles que residem com seus cônjuges. Porém, há indícios que de uma inclinação dos usuários residirem com familiares/parentes (MELQUIADES R, et al., 2016). Mas, outros achados sinalizam o contrário, pré-dispondo que, majoritariamente, os portadores de DRC moram com seus companheiros/cônjuges (HALL RK, et al., 2018).

Alusivo à religião que os usuários referiram seguir, nota-se associação estatística com o catolicismo, corroborando assim, com outras pesquisas (KIM JY, et al., 2013). Contudo, um estudo com a finalidade de pesquisar desesperança, ideação suicida e depressão, desvelam que não há associação entre o catolicismo e a DRC (AGUIAR LK, et al., 2020). Nesse aspecto, outras buscas admitem uma correlação entre a boa qualidade de vida e um alto nível de religiosidade, reforçando a positividade da fé individual (GOLÇALVES FA, et al., 2015).

A HAS é apontada em alguns estudos como principal patologia de base da DRC (MONTEIRO MAC, et al., 2018) Pesquisas destacam essa estreita relação entre HAS, DM e a DRC, sendo as principais doenças de base para seu surgimento. Outras patologias, como glomerulonefrites, rins policísticos e outras causas desconhecidas, estiveram presentes nesses estudos, mas de forma amena (MARQUES VR, et al., 2016; GESUALDO GD, et al., 2016).

Consoante ao tempo em que os usuários estão em tratamento de hemodiálise, através do teste realizado, não se pode assinalar que a DRC está mais vigente em uma determinada faixa de tempo de tratamento. Estes achados não estão similares a alguns resultados encontrados por outros autores (MIYAHIRA CK, et al., 2016; VENTURA J, et al., 2018). Por outro lado, há prenúncios que inferem resultados semelhantes, testificando em seu estudo que o tempo mais prevalente gira em torno de $\geq 2 \mathrm{e} \leq 5$ anos de tratamento (OLIVEIRA APB, et al., 2016).

Ao refletir-se sobre as variáveis que evidenciaram significância, é necessária atenção aos homens. É sabido que os homens procuram menos os serviços de saúde, essa situação somado à uma maior falta de interesse no autocuidado, estão mais sujeitos a serem acometidos pelas doenças crônicas, o que revela um fator de risco para o surgimento da DRC em indivíduos do sexo masculino (MONTEIRO MAC, et al., 2018).

Além disso, os homens casados aparentam ter um fator de proteção em relação aos solteiros, visto que os casados têm o amparo da companheira ou filhos que muitas vezes o estimulam a buscar assistência em saúde precocemente. Diferentemente, os solteiros tendem a buscar ajuda mais tardiamente, uma vez que são menos estimulados e acabam sendo influenciados de forma negativa por outros solteiros (SARTORI A, et al., 2017).

Verifica-se características que evidenciam vulnerabilidades à baixa escolaridade, sendo esta, um fator que limita as oportunidades de emprego, situação que pode justificar a baixa renda. É importante destacar que estar em um tratamento hemodialítico por si só representa um aspecto que interfere na realização de atividades laborais. É necessária uma rotina rígida de tratamento para manutenção de saúde, podendo prejudicar continuidade dos estudos e produtividade no trabalho. 
Em relação às médias dos escores obtidos através do KDQOL-36 $6^{\mathrm{TM}}$, percebe-se que os efeitos da doença renal interferem de maneira considerável na QV dos usuários, tal relação, dá-se por haver diversas mudanças relacionadas à alimentação, restrição de líquidos, diminuição da capacidade laboral, estresse, preocupações com a doença renal e aparência (OTONI A, et al., 2019).

Nesse aspecto, é possível observar que a média da dimensão do componente físico apresenta baixo escore $(39,5)$ quando comparado ao componente mental $(45,1)$, tais resultados corroboram com estudo já realizado, os quais evidenciam que usuários em terapia hemodialítica deixam de exercer atividades laborais e instrumentais, em decorrência das limitações que a DRC causa ao corpo do indivíduo, levando a um baixo rendimento físico, o que, inevitavelmente, reflete na sua QV (KIM JY, et al., 2013).

Para além disso, o domínio da carga que a DRC agrega na vida do usuário, mostra-se com significativo comprometimento na QV, explicado o baixo escore $(36,4)$; esses achados são semelhantes em outras literaturas, as quais mostram usuários decepcionados como a sua DRC, assim como frustrados com o peso que a doença traz à sua vida, a ponto de muitos declararem sentirem-se um peso para sua família, evidenciando que as dimensões psicológicas do usuário também são afetadas dolorosamente (OLIVEIRA et al., 2016; MARINHO DF, et al., 2020).

A correlação estatística entre as dimensões possibilita entender que, na medida em que uma dimensão denota baixo escore induz outra a também se inclinar para valores baixos. As dimensões mostraram-se interligadas e uma inspira a outra, evidenciando o impacto na QV. O inverso disso também ocorre, por exemplo: se a dimensão sintomas da DRC expressar níveis bons de QV, refletirá positivamente na dimensão dos efeitos da DRC na vida diária do usuário.

Os itens do KDQOL-36 ${ }^{\mathrm{TM}}$ conectam-se revelando que o componente mental afeta o componente físico e vice-versa. Assim como os dois refletem e são refletidos pelos sintomas da DRC. Já o componente mental atinge e é atingido pela carga da DRC; semelhantemente, os sintomas da DRC incidem e são incididos pelos efeitos da DRC e carga da DRC. Essas correlações explicam linearmente suas influências na QV dos doentes renais.

Nessa conjuntura, os itens que fazem parte do componente físico, tratam-se de restrições impostas pela DRC na vida do doente, isto é, o usuário perde a capacidade de realizar atividades mais pesadas ou leves que necessitam de um desempenho físico, dessa forma, provocando frustrações mentais e sentimentos negativos como tristeza, solidão e isolamento; daí, explica-se a relação de interferências entre as duas dimensões (VENTURA J, et al., 2018).

Nesse sentido, ocorrem relações parecidas entre: componentes físicos e sintomas da DRC e entre componentes mentais e sintomas da DRC. Dado que, na medida em que os sintomas da DRC (dor, náusea, cãibras, esgotamento físico, falta de ar etc.) aparecem e intensificam-se no individuo, necessariamente, haverá uma reflexão negativa na esfera física e mental do usuário (MENEZES HF, et al.,2017).

Assim como há íntima relação entre a carga da DRC e componentes mentais; sintomas das DRC e carga da DRC; e, sintomas da DRC e efeitos da DRC na vida diária do usuário. Porquanto a carga da DRC está atrelada a uma percepção pessoal de angustia mental, sendo que na medida em que os sintomas surgem e se agravam na vida do portador da DRC, essa carga de portar a doença torna-se mais pesada; dessa forma, os sintomas da doença estão interligados com os efeitos que a mesma causa na vida do usuário, uma vez que esses efeitos (restrição de líquida e alimentar, sexo, capacidade de viajar etc.) surgem a partir dos sintomas e prejuízos que a patologia acresce (MARQUES VR, et al., 2016).

Para entender melhor os motivos pelos quais há essa teia apontada através das correlações estatísticas entre as dimensões do KDQOL-36 ${ }^{\mathrm{TM}}$ e, nas particularidades do indivíduo, é necessário que se recorra a fundamentos que predispõem que o ser humano deve ser observado como um ser holístico, ou seja, como um todo, corpo, mente, espírito e emoções. Assim, toda e qualquer forma de abordagem e análise em relação ao indivíduo deve ser pautado no saber de estar lidando com um ser biopsicossocial (GESUALDO GD, et al., 2016). 


\section{CONCLUSÃO}

O estudo sinaliza que os mais acometidos pela DRC são solteiros, os que ganham entre 1 e 3 salários mínimos, aposentados e católicos. Além disso, reforça que os usuários portadores de DRC não têm uma QV satisfatória e, além disso, em algumas dimensões, como já observado, os escores de QV são baixos, evidenciando que os aspectos biopsicossociais dessas pessoas estão sendo prejudicados. Não obstante, os frutos desse estudo apresentam subsídios para que a equipe multidisciplinar de saúde reconheça a importância de descobrir as complicações/sentimentos pertencentes à vida do doente renal que faz hemodiálise, bem como a interferência dessa particularidade terapêutica na vida da pessoa.

\section{REFERÊNCIAS}

1. AGUIAR LK, et al. Fatores associados à doença renal crônica: inquérito epidemiológico da Pesquisa Nacional de Saúde. Revista Brasileira de Epidemiologia, 2020; 23(1): e200044.

2. ASLAN I. Aplicação do formulário KDQOL TM -36 em pacientes com DRC. Revista de Tópicos Atuais em Gestão em Saúde (COES \& RJ-JHM), 2018; 1(1): 11-21.

3. BEBER GC, et al. Qualidade de vida de pacientes transplantados renais após longo período do transplante. Saúde e Pesquisa, 2017; 10(1): 163-170.

4. CRUZ MRF, et al. Descoberta da doença renal crônica e o cotidiano da hemodiálise/Discovery of chronic kidney disease and every day of hemodialysis. Ciência, Cuidado e Saúde, 2016; 15(1): 36-43.

5. GESUALDO GD, et al. Fatores associados à fragilidade de idosos com doença renal crônica em hemodiálise. Ciênc. saúde coletiva, Rio de Janeiro, 2016; 21(11): 3493-3498.

6. GONÇALVES FA, et al. Qualidade de vida de pacientes renais crônicos em hemodiálise ou diálise peritoneal: estudo comparativo em um serviço de referência de Curitiba-PR. Brazilian Journal of Nephrology, 2015; 37(4): 467-474.

7. HALL RK, et al. Associação da Qualidade de Vida na Doença Renal (KDQOL-36 ${ }^{\mathrm{TM}}$ ) com mortalidade e hospitalização em idosos em hemodiálise. BMC nefrology, 2018; 19(1): 11.

8. KIM JY, et al. Health-related quality of life with KDQOL-36 $6^{\mathrm{TM}}$ and its association with self-efficacy and treatment satisfaction in Korean dialysis patients. Quality of Life Research, 2013; 22(4): 753-758.

9. MARINHO DF, et al. Capacidade funcional e qualidade de vida na doença renal crônica. Revista Pesquisa em Fisioterapia, 2020; 10(2): 212-219.

10. MARQUES VR, et al. Avaliação da intensidade da dor de pacientes renais crônicos em tratamento hemodialítico. Revista Dor, 2016;17(2): 96-100.

11. MELQUIADES R, et al. Fístula arteriovenosa na perspectiva de pacientes renais crônicos. Enferm. Foco [Internet], $2016 ; 7(1): 37-41$.

12. MENEZES HF, et al. Características sociodemográficas, clínicas e subjetivas de clientes com doença renal crônica atendidos na consulta de enfermagem. Rev. enferm. UFPE on line, p. 1858-1866, 2017.

13. MIYAHIRA CK, et al. Avaliação da dor torácica, sono e qualidade de vida de pacientes com doença renal crônica. Arquivos de Ciências da Saúde, 2016; 23(4): 61-66.

14. MONTEIRO MAC, et al. Chronic renal disease: characteristics of patients waiting for renal transplantation/Doença renal crônica: características dos pacientes que aguardam o transplante renal/Enfermedad renal crónica: características de los pacientes que aguar. Revista de Enfermagem da UFPI, 2018; 7(2): 18-22.

15. OLIVEIRA APB, et al. Quality of life in hemodialysis patients and the relationship with mortality, hospitalizations and poor treatment adherence. Brazilian Journal of Nephrology, 2016; 38(4): 411-420.

16. OTONI A, et al. - Domínios de qualidade de vida mais prevalentes entre pacientes em hemodiálise e diálise peritoneal. Ciência ET Praxis, 2019; 12(24): 13-22.

17. PEIPERT JD, et al. Qualidade normativa da Doença Renal 36-Item Short Form Survey (KDQOL-36 ${ }^{\mathrm{TM}}$ ) valores normativos para a população de diálise dos Estados Unidos e nova pontuação única e resumida. Jornal da Sociedade Americana de Nefrologia, 2019; 30(4): 654-663.

18. PEREIRA RMP, et al. Qualidade de vida de idosos com doença renal crônica em tratamento conservador. Revista Brasileira de Enfermagem, 2017, 70:4.

19. RIBEIRO KRA. Cuidados de enfermagem aos pacientes com insuficiência renal crônica no ambiente hospitalar. Revista Recien-Revista Científica de Enfermagem [internet]: 2016; 6(18): 26-35

20. SANTOS BP, et al. Doença renal crônica: relação dos pacientes com a hemodiálise. ABCS health sci, 2017; 24(4): 8-14.

21. SARTORI A, et al. Impacto do cronotipo na qualidade de vida de pacientes renais crônicos submetidos a tratamento hemodialítico. Ciência \& Saúde, 2017; 10(3): 161-169.

22. VENTURA J, et al. Pacientes em tratamento hemodialítico: percepção acerca das mudanças e limitações da doença e tratamento. Rev. pesqui. cuid. fundam. (Online), 2018; 6(1): 926-931. 